\title{
MODELO DE INFECCIÓN EXPERIMENTALPARA LA OBTENCIÓN DE E. granulosus ADULTOS Y QUISTES HIDATÍDICOS SECUNDARIOS EN EL HÁMSTER DORADO (Mesocricetus auratus)
}

\author{
EXPERIMENTAL INFECTION Model to ObTaIN E. gRANULOSUS AdULT Forms AND \\ Secondary Hydatidic Cystic in the Golden Hamster (Mesocricetus auratus)
}

\author{
Gonzalo Vallenas R. ${ }^{1}$, Jéssica Alvarado G. ${ }^{1}$, Eduardo Barrón G. ${ }^{1}$, Rosa Perales C. ${ }^{2}$,
} César Gavidia C.,3

\section{Resumen}

\begin{abstract}
El objetivo del estudio fue evaluar un modelo animal alternativo al uso de caninos como hospedero definitivo y al uso de ovinos como hospedero definitivo de Echinococcus granulosus. Se utilizaron 33 hámster dorados (Mesocricetus auratus) hembras. El grupo A, conformado por 16 hámster de un mes de edad fue infectado, vía oral, con 3000 protoescólices viables de E. granulosus por animal; y el grupo B, conformado por 17 hámster de 4.5 meses de edad fue infectado, vía intraperitoneal, con 1500 protoescólices viables por animal. Todos los protoescólices provenían de quistes fértiles de ovinos naturalmente infectados. No se logró obtener E. granulosus adultos en los animales del grupo A, mientras que en el grupo B se obtuvo quistes hidatídicos secundarios en 9 animales (52.9\%) a los 2.5, 3.5 y 6.5 meses post-infección; sin embargo, todos los quistes obtenidos fueron infértiles.
\end{abstract}

Palabras clave: modelo animal, hámster dorado, Echinococcus granulosus, hidatidosis, protoescólices, infección experimental

\section{Abstract}

The objective of the study was to evaluate an alternative animal model to the use of canines as definitive host and the use of sheep as definitive host of Echinococcus granulosus. Thirty three female Golden hamsters (Mesocricetus auratus) were used. In group A, 16 animals of one month of age were orally infected with 3000 viable protoscoleces of E. granulosus; and in group B, 17 animals of 4.5 months of age were intraperitoneal infected with 1500 viable protoscoleces. All protoscoleces were obtained from fertile cysts collected from naturally infected sheep. No adult forms of E. granulosus were

\footnotetext{
${ }^{1}$ Laboratorio de Medicina Veterinaria Preventiva, ${ }^{2}$ Laboratorio de Histología, Embriología y Patología Veterinaria, Facultad de Medicina Veterinaria, Universidad Nacional Mayor de San Marcos, Lima

${ }^{3}$ E-mail: cmgavidia@yahoo.com
} 
recovered from group A, while in group B secondary hydatid cysts were recovered in 9 animals (52.9\%) at 2.5, 3.5 and 6.5 months after infection. All recovered cysts were infertile.

Key words: animal model, golden hamster, Echinococcus granulosus, hydatidosis, protoscoleces, experimental infection

\section{INTRODUCCIÓN}

La hidatidosis, enfermedad producida por la fase larvaria de Echinococcus granulosus, ha sido reconocida como uno de los mayores problemas de salud pública en el mundo (Moro et al, 1999; Dueger y Gilman, 2001). En el Perú, afecta a gente de escasos recursos económicos, sobre todo en áreas de alta endemicidad, como la Sierra Sur y Sierra Central de los Andes (Rojas, 2004; Moro y Schantz, 2006).

Se han realizado diversos trabajos de investigación utilizando al perro como animal de experimentación (Lopera, 1998), pero la inversión en alimento, alojamiento, personal y escasez de animales representan una seria limitante; asimismo, el uso de ovinos como animales de experimentación es aún más costoso y requiere de mayor apoyo logístico, de allí la necesidad de buscar otras alternativas de modelos animales que resulten más ventajosas.

Los modelos experimentales realizados en animales de laboratorio han servido para evaluar el comportamiento de varias enfermedades que afectan principalmente al hombre (Zúñiga et al., 2001). Estos modelos animales permiten, en algunos casos, la extrapolación de los resultados a la especie humana y a los animales de interés económico (Zúniga et al., 2001). García et al. (1997) lograron obtener quistes hidatídicos secundarios a partir de la infección intraperitoneal de protoescólices en el $100 \%$ de gerbillos (Meriones unguiculatus) infectados. En forma similar, Zhang et al. (2005) consiguieron quistes hidatídicos secundarios en todos los ratones infectados y protoescólices en muchos de estos quistes reproducidos en ratones.

Los modelos experimentales realizados en animales de laboratorio son una buena alternativa de hospedero definitivo e intermediario para E. granulosus, permitiendo explicar y determinar la probable existencia de cepas del parásito. Así, por ejemplo se han encontrado diferencias morfológicas y bioquímicas en parásitos adultos (Denegri et al., 2002). Por otro lado, también se podría considerar tratamientos farmacológicos contra las formas larvarias o quistes.

El objetivo del presente trabajo fue evaluar al hámster como un potencial modelo animal para la obtención del estadio adulto y larvario (quiste hidatídico) de Echinococcus granulosus.

Materiales y Métodos

\section{Ubicación del Estudio}

El estudio se realizó en el Laboratorio de Medicina Veterinaria Preventiva de la Facultad de Medicina Veterinaria, Universidad Nacional Mayor de San Marcos, Lima.

\section{Protoescólices}

Los protoescólices (PE) utilizados para la infección experimental procedieron de quistes hidatídicos fértiles de pulmón de ovinos naturalmente infectados del departamento de Ayacucho, y que fueron sacrificados en el camal local. El tiempo transcurrido entre la 
colección de los quistes y su procesamiento fue de 1 a 2 horas. Una vez obtenido el órgano con el quiste hidatídico, se desinfectó con algodón embebido en alcohol la zona del quiste a ser punzada. Con una jeringa hipodérmica se extrajo y se devolvió el líquido quístico tres veces como mínimo a fin de homogenizar el contenido y facilitar la extracción de los PE contenidos en el quiste. Luego, el líquido extraído se depositó en tubos falcon de $50 \mathrm{ml}$, se dejó en reposo a $4{ }^{\circ} \mathrm{C}$ con ayuda de refrigerantes por espacio de 20 minutos, y se retiró el sobrenadante dejando un volumen de $10 \mathrm{ml}$. En todo momento, los tubos falcón conteniendo los PE estuvieron en contacto con refrigerantes para disminuir la cantidad de PE muertos o evaginados.

La viabilidad de los PE se determinó con el método de exclusión de tinciones vitales, en el cual se utiliza una solución de eosina al $0.1 \%$ (Himonas et al., 1994). Los PE que se tiñen con la eosina están muertos y los que mantienen su color o no se tiñen están vivos. El porcentaje de viabilidad se calculó con la siguiente fórmula: (PE vivos sin evaginar $\mathrm{x}$ 100)/PE totales.

Los PE fueron mantenidos en su propio líquido o agregando solución salina al 0.9\% y fueron utilizados en forma inmediata para realizar las infecciones experimentales. El número total de protoescólices vivos sin evaginar de la muestra se calculó en base al número de $\mathrm{PE}$ encontrados en el conteo en una lámina $(50 \mu \mathrm{l})$, separándose el volumen requerido para cada animal a desafiar. El tiempo transcurrido hasta la infección de los animales fue de aproximadamente dos horas.

\section{Animales Experimentales}

Se utilizaron 33 hámster dorados (Mesocricetus auratus), de sexo hembra y en aparente buen estado de salud, procedentes del Instituto Nacional de Salud, Lima, Perú. Los animales fueron desparasitados el día de su recepción con praziquantel en dosis de $25 \mathrm{mg} / \mathrm{kg}$ p.v. y al día siguiente con albendazol en dosis de $25 \mathrm{mg} / \mathrm{kg}$ p.v., en ambos casos por vía oral (Sumano y Camberos, 1997; Adams, 2003). Se les mantuvo en jaulas estándares para roedores de laboratorio, donde recibieron alimento concentrado comercial para hámsteres y agua ad libitum. Todos tuvieron un periodo de descanso, aclimatación y cuarentena por 10 días antes de proceder con las inoculaciones.

Dieciséis hámster de un mes de edad fueron infectados por vía oral, y 17 individuos de 4.5 meses de edad fueron infectados por vía intraperitoneal. Los animales del grupo de infección oral fueron inmunosuprimidos con la administración de acetato de metilprednisolona (Depomedrol) a una dosis de $2 \mathrm{mg}$ /animal, vía i.m., un día antes de la infección y semanalmente después de la infección hasta completar el periodo prepatente - 48 a 52 días - del parásito (Lapage, 1983; Soulsby, 1987; Atías, 1994; Cordero del Campillo et al, 1999; Barriga, 2002; Acha y Szyfres, 2003).

Para el sacrificio, los animales fueron anestesiados con una dosis combinada de ketamina $(10 \mathrm{mg} / \mathrm{kg})$ y xilacina $(10 \mathrm{mg} / \mathrm{kg})$, vía i.m. Se les tomó una muestra de sangre y se procedió al sacrificio mediante desnucamiento. Los animales fueron manejados bajo las normas internacionales sobre el uso de animales de laboratorio para minimizar al máximo el sufrimiento de los mismos y brindarles el bienestar adecuado durante el desarrollo del estudio.

\section{Infección Experimental}

Infección oral. Cada animal $(\mathrm{n}=16)$ recibió $3000 \mathrm{PE}$ vivos a través de una jeringa de tuberculina y fueron sacrificados al término del periodo prepatente del parásito. Luego del sacrificio, se extrajo el intestino delgado y se le dividió en tres secciones: anterior, media y posterior, según el estudio de Lopera (1998). Cada sección fue colocada en una placa petri con suero fisiológico, se abrió el intestino y se procedió al raspado de la mucosa. La búsqueda, contaje, medición y observación de 
Cuadro 1. Hámster dorados que fueron infectados con 1500 protoescólices de $E$. granulosus, vía intraperitoneal, y que desarrollaron quistes hidatídicos secundarios

\begin{tabular}{ccccc}
\hline Animal & $\begin{array}{c}\text { Meses post- } \\
\text { infección }\end{array}$ & $\begin{array}{c}\text { Número de } \\
\text { quistes }\end{array}$ & $\begin{array}{c}\text { Víscera } \\
\text { afectada }\end{array}$ & $\begin{array}{c}\text { Tamaño } \\
(\mathrm{mm})\end{array}$ \\
\hline 1 & 2.5 & 1 & Hígado & $5 \times 4$ \\
2 & 2.5 & 1 & Hígado & $1 \times 1$ \\
5 & 3.5 & 2 & Hígado & $3 \times 1$ \\
6 & 3.5 & 1 & Hígado & $1 \times 1$ \\
7 & 3.5 & 1 & Pulmón & $4 \times 3$ \\
9 & 6.5 & 2 & Hígado & $2 \times 3$ \\
11 & 6.5 & 1 & Hígado & $2 \times 3$ \\
14 & 6.5 & 1 & Hígado & $14 \times 16$ \\
16 & 6.5 & 1 & Hígado & $10 \times 10$ \\
\hline
\end{tabular}

proglótidos de E. granulosus se hizo con ayuda de un esterocopio.

Infección intraperitoneal. Cada animal $(\mathrm{n}=17)$ fue infectado con una dosis de 1500 PE vivos a través de una jeringa de tuberculina. Para la inyección se tomó como punto de referencia el punto medio imaginario existente entre la cicatriz umbilical y el cartílago xifoides. Seis, seis y cinco animales fueron sacrificados a los 2.5, 3.5 y 6.5 meses post-infección, respectivamente. Se observó durante la necropsia la presencia de quistes hidatídicos secundarios en hígado y pulmón, y si fuese el caso en otros órganos. Se tuvo en cuenta la localización, tamaño, forma y número de quistes, así como las características del líquido hidatídico. El tamaño de los quistes (largo x ancho) se midió usando un calibrador Bernier. Los órganos de animales positivos o sospechosos de presentar quistes hidatídicos fueron conservados en formalina $10 \%$ y remitidos al laboratorio de histopatología para el estudio respectivo. Se empleó la tinción de hematoxilina-eosina en los cortes histológicos.

\section{Resultados y Discusión}

No se logró observar E. granulosus adultos en ninguna de las tres secciones del intestino delgado de hámsteres infectados por vía oral; resultado contradictorio con los obtenidos por Conchedda et al. (2006) quienes lograron reproducir E. granulosus adultos en intestino delgado de gerbillos (Meriones unguiculatus), posiblemente por haber considerado en ese estudio la actividad de las células flamígeras y la activación de los protoescólices. Al parecer, la inmunosupresión de los hámster con corticoides y el criterio de exclusión de tinciones vitales, no fueron suficientes para lograr el desarrollo de $E$. granulosus en el intestino delgado. Además, es posible que el hámster dorado, hospedero no innato, no sea susceptible a este parásito, de modo que el intestino delgado no reúna las características adecuadas para el crecimiento y maduración del E. granulosus, más allá de que los animales fueron inmunosuprimidos. 
En el caso de los 17 hámster dorados infectados por vía intraperitoneal, nueve resultaron positivos a quistes hidatídicos (Cuadro 1). Los animales positivos a los 2.5 meses post-infección presentaron los quistes hidatídicos secundarios de 1 y $5 \mathrm{~mm}$ de largo en el hígado con un promedio de $0.1 \mathrm{ml}$ de líquido hidatídico de apariencia transparente (Fig. 1a).

Dos de los tres animales positivos a los 3.5 meses post-infección presentaron quistes hidatídicos secundarios en hígado y el tercero en el pulmón. El tamaño de los quistes varió entre 1 y $3 \mathrm{~mm}$ con $0.25 \mathrm{ml}$ de líquido hidatídico en promedio (Fig. 1b). Asimismo, todos los quistes a los 6.5 meses post-infec- ción se presentaron en el hígado, con un tamaño entre 2 y $16 \mathrm{~mm}$ y con un contenido líquido de $0.4 \mathrm{ml}$ (Fig. 1c). Todos los quistes hidatídicos secundarios en los nueve animales positivos fueron infértiles, es decir, negativos a la presencia de protoescólices.

En el examen histopatológico de los quistes, se observa claramente el epitelio y en algunos casos la arenilla hidatídica y mucopolisacáridos, probablemente originados por el metabolismo del parásito. También se observa reacción inflamatoria con aglomerados de linfocitos e histiocitos (Fig. 2), compatibles con otros resultados (Yamashita et al., 1956, 1960).

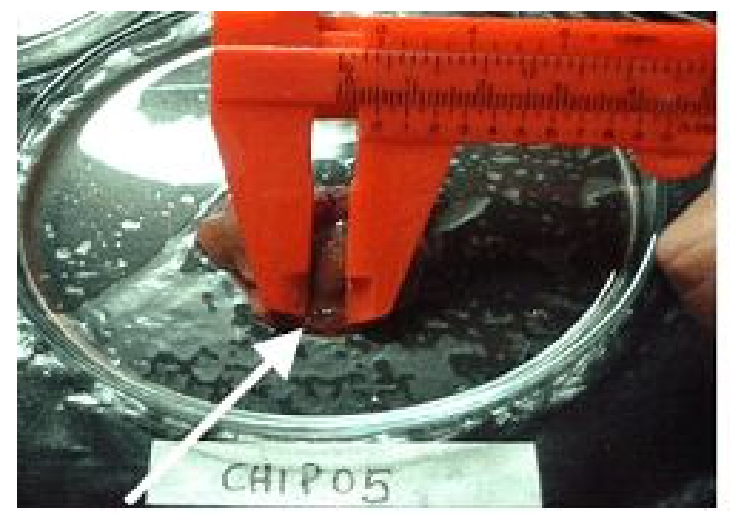

(a)

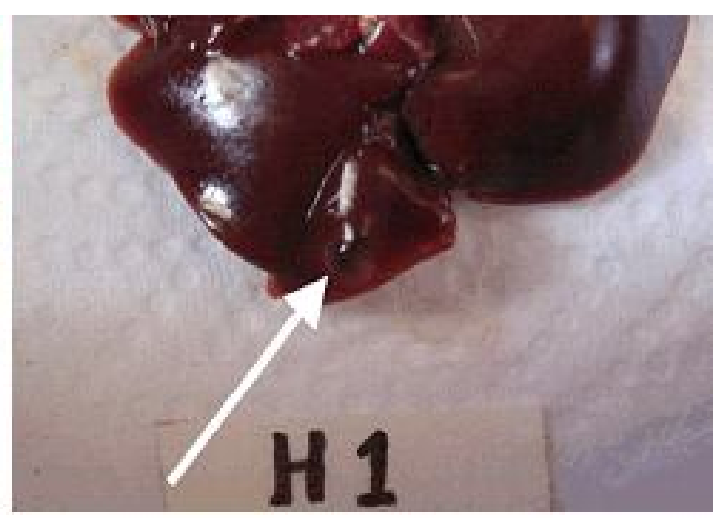

(b)

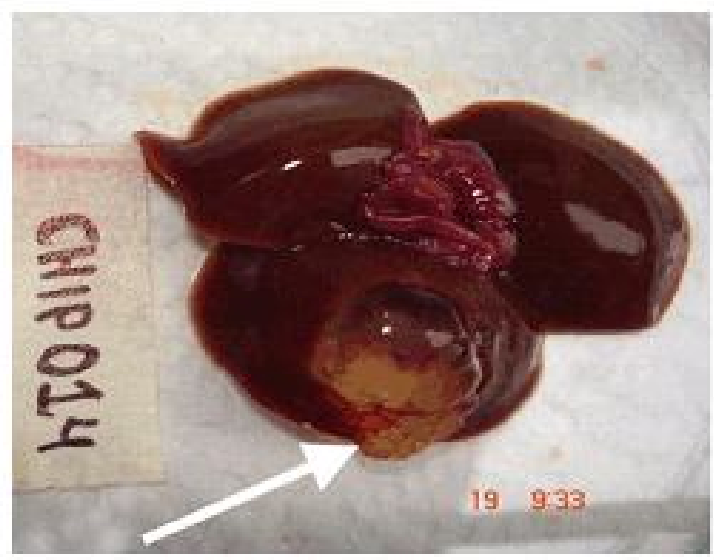

(c)

Figura 1. Imágenes macroscópicas de quistes hidatídicos secundarios en hámster dorado. a) A los 2.5 meses post-infección; b) A los 3.5 meses post-infección; c) A los 6.5 meses post-infección 


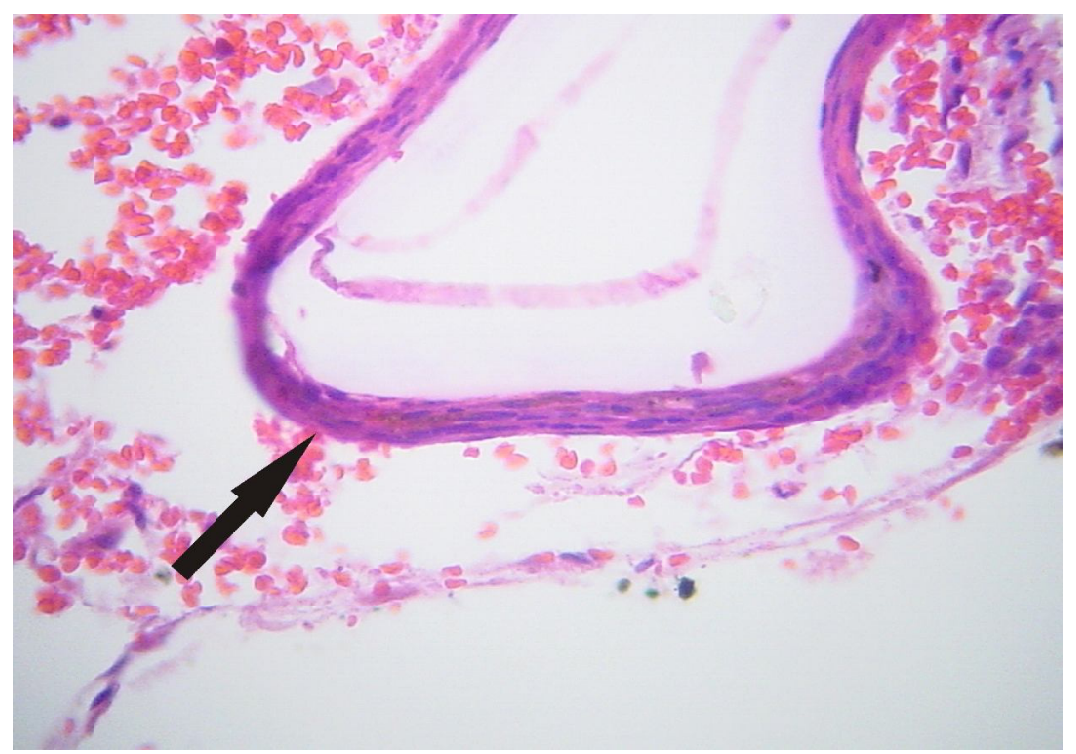

Figura 2. Diagnóstico histopatológico. Corte de hígado de hámster dorado. Cavidad quística formada por un epitelio plano estratificado, donde se aprecia una estructura celular acidófila en el centro que parece haberse desprendido de la membrana del quiste (HE, 100X).

Los resultados muestran que la infección intraperitoneal con protoescólices de $E$. granulosus en hámster dorados produjeron quistes hidatídicos secundarios en 9 de los 17 animales infectados (52.9\%), resultado inferior a un estudio en gerbillos (Meriones unguiculatus), donde se logró reproducir quistes hidatídicos secundarios en todos los animales entre los 2.5 a 12 meses post- infección utilizando la misma cantidad de protoescólices de E. granulosus (García et al., 1997). Los resultados obtenidos tampoco coinciden con los de Zhang et al. (2005) en ratones $\mathrm{BALB} / \mathrm{c}$, donde los protoescólices fueron cultivados in vitro por 56 días produciendo pequeños quistes que fueron empleados para infectar ratones. Es posible que las diferencias encontradas se deban a los criterios utilizados para medir la viabilidad de los protoescólices, ya que en el estudio de García et al. (1997) se consideró, además de los criterios empleados en el presente estudio, la presencia de ganchos rostelares intactos, la actividad de las células flamígeras y el número de corpúsculos calcáreos. Estos últimos podrían, al parecer, aumentar la probabilidad de supervivencia en un medio que no reúne las características necesarias para su desarrollo (Holeman y Heath, 1997).

\section{Conclusiones}

El modelo experimental utilizando al hámster dorado resultó adecuado para obtener quistes hidatídicos secundarios mediante la inoculación por vía intraperitoneal de protoescólices viables, aunque infértiles, de E. granulosus de pulmón ovino. Sin embargo, no logró reproducir E. granulosus adultos en el intestino delgado mediante la inoculación por vía oral de los protoescólices viables. 


\section{Literatura Citada}

1. Acha P, Szyfres B. 2003. Zoonosis y enfermedades transmisibles comunes al hombre y a los animales. Vol 3. $3^{\mathrm{a}}$ ed. Washington DC: Organización Panamericana de la Salud. 380 p.

2. Adams R. 2003. Farmacología y terapéutica veterinaria. $2^{\circ}$ ed. Ed. Zaragoza: Acribia. $1281 \mathrm{p}$.

3. Atías A. 1994. Parasitología clínica. $3^{\circ}$ ed. Santiago de Chile: Publ Téc Mediterráneo. 234 p.

4. Barriga O. 2002. Las enfermedades parasitarias de los animales domésticos en la América Latina. Santiago de Chile: Ed Germinal. 247 p.

5. Conchedda M, Gabriele F, Bortoletti C. 2006. Development and sexual maturation of Echinococcus granulosus adult worms in the alternative definitive host, Mongolian gerbil (Meriones unguiculatus). Acta Trop 97: 119-125.

6. Cordero del Campillo M, Rojo F, Martínez A, Sánchez M, Hernández S, Navarrete I, Diez P, et al. 1999. Parasitología veterinaria. Madrid: Mc Graw-Hill Interamericana. 968 p.

7. Denegri G, Elisondo M, Dopchiz M. 2002. Situación de la hidatidosisequinococosis en la República Argentina. Mar del Plata: Ed Martín. 244 p.

8. Dueger E, Gilman R. 2001. Prevalence, intensity and fertility of ovine cystic echinoccocosis in the central Peruvian Andes. T Roy Soc Trop Med H 95: 379-383.

9. García L, Álvarez A, Redondo P, Prieto J. 1997. Estudio de la fertilidad y viabilidad de quistes hidatídicos ovinos. Rev Esp Salud Púb 71(5): 25-30.

10. Himonas C, Antoniadou-Sotiriodou K, Papadopoulos E. 1994. Hidatidosis of food animals in Greece: prevalence of cysts containing viable protoscoleces. $\mathbf{J}$ Helminthol 68: 311-313.
11. Holeman B, Heath D. 1997. The early stages of Echinococcus granulosus development. Acta Trop 64: 5-17.

12. Lapage G 1983. Parasitología veterinaria. México DF: Ed Continental. 483 p.

13. Lopera L. 1998. Diagnóstico de la equinococosis canina mediante la purga con arecolina y la prueba de ELISA para la detección de coproantígeno. Lima: Tesis de Médico Veterinario. Univ Nacional Mayor de San Marcos. 49 p.

14. Moro P, Schantz P. 2006. Cystic echinococcosis in the Americas. Parasitol Int 55: 5181-5186.

15. Moro P, Mc Donald J, Gilman R, Silva B, Verástegui M, Malqui V, Lescano $G$, et al. 1999. Epidemiology of Echinococcus granulosus infection in Peruvian Andes. B World Health Organ 75: 553-561.

16. Rojas M. 2004. Nosoparasitosis de los rumiantes domésticos peruanos. Lima: Martegraf. $146 \mathrm{p}$.

17. Soulsby E. 1987. Parasitología y enfermedades parasitarias de los animales domésticos. $7^{\circ}$ ed. México DF: Nueva Ed Interamericana. $823 \mathrm{p}$.

18. Sumano H, Camberos L. 1997. Farmacología veterinaria. $2^{\circ}$ ed. México DF: Mc Graw-Hill Interamaericana. $680 \mathrm{p}$.

19. Yamashita J, Ohbayashi M, Cono S. 1956. Studies on echinococcosis. IV. Experimental infection of the white mouse. Jap J Vet Res 4: 122-129.

20. Yamashita J, Ohbayashi M, Sakamoto T. 1960. Studies on echinococcosis. XI. Observations on secondary echinococcosis. Jap J Vet Res 8: 314-323.

21. Zhang W, Jones M, Li J, Mc Manus D. 2005. Echinococcus granulosus. Pre-culture of protoscoleces in vitro signifícantly increases development and viability of secondary hydatid cysts in mice. Exp Parasitol 110: 88-90.

22. Zúñiga J, Tur J, Milocco S, Piñeiro R. 2001. Ciencia y tecnología en protección y experimentación animal. Madrid: Mc Graw Hill-Interamericana. 683 p. 